\title{
Genetic variations of ND5 gene of mtDNA in populations of Anopheles sinensis (Diptera: Culicidae) malaria vector in China
}

\author{
Abdelrafie M Makhawi ${ }^{1,2,3,4}$, Xiao-Bo Liu ${ }^{1,2,3,5}$, Shu-Ran Yang ${ }^{1,2,3}$ and Qi-Yong Liu 1,2,3,5*
}

\begin{abstract}
Background: Anopheles sinensis is a principal vector for Plasmodium vivax malaria in most parts of China. Understanding of genetic structure and genetic differentiation of the mosquito should contribute to the vector control and malaria elimination in China.

Methods: The present study investigated the genetic structure of An. sinensis populations using a 729 bp fragment of mtDNA ND5 among 10 populations collected from seven provinces in China.

Results: ND5 was polymorphic by single mutations within three groups of An. sinensis that were collected from 10 different geographic populations in China. Out of 140 specimens collected from 10 representative sites, 84 haplotypes and 71 variable positions were determined. The overall level of genetic differentiation of An. sinensis varied from low to moderate across China and with a $F_{\text {ST }}$ range of $0.00065-0.341$. Genealogy analysis clustered the populations of An. sinensis into three main clusters. Each cluster shared one main haplotype. Pairwise variations within populations were higher (68.68\%) than among populations (31.32\%) and with high fixation index $\left(F_{S T}=0.313\right)$. The results of the present study support population growth and expansion in the An. sinensis populations from China. Three clusters of An. sinensis populations were detected in this study with each displaying different proportion patterns over seven Chinese provinces. No correlation between genetic and geographic distance was detected in overall populations of $A$. sinensis $\left(R^{2}=0.058 ; P=0.301\right)$.

Conclusions: The results indicate that the ND5 gene of mtDNA is highly polymorphic in An. sinensis and has moderate genetic variability in the populations of this mosquito in China. Demographic and spatial results support evidence of expansion in An. sinensis populations.
\end{abstract}

Keywords: Anopheles sinensis, Population genetics structure, mtDNA, ND5 gene

\section{Background}

Anopheles sinensis is a principal vector of malaria in many regions in China along with An. lesteri, An. minimus, and An. dirus [1,2]. An. sinensis has also been identified as the major malaria vector in many parts of other Asian countries, such as in Indonesia, South Korea, Thailand and Japan [3]. In China it was reported that $0.006-2.58 \%$ of

\footnotetext{
* Correspondence: liuqiyong@icdc.cn

'Department of Vector Biology and Control, State Key Laboratory for Infectious Disease Prevention and Control, National Institute for Communicable Disease Control and Prevention, Chinese Center for Disease Control and Prevention, Beijing 102206, China

${ }^{2}$ WHO Collaborating Centre for Vector Surveillance and Management, Beijing 102206, China

Full list of author information is available at the end of the article
}

An. sinensis was infected with $P$. vivax. The infection rate of An. sinensis in Taiwan was $0.02 \%$ during 1947-1949 [4]. However, malaria outbreaks and re-emergences in China occurred only in the areas where An. sinensis is the principal vector in recent years [5]. Malaria was historically epidemic in the Huang-Huai River region of central China and the total malaria cases in these areas were 21.99 million, accounting for $91.2 \%$ of the total reported cases in the country in 1970s. With active implementation of malaria control measures for more than 30 years, considerable success had been achieved and the cases decreased dramatically and many counties in Huang-Huai River region reached the goals of the malaria elimination (the incidence is below $1 / 10,000)$. However, in the past decade, malaria has re-

\section{Biomed Central}


emerged in these areas, especially the Anhui Province, where a total of 26,873 malaria cases, 108,594 suspected cases and 23 deaths were reported by the annual case reporting system in 858 counties of 22 Provinces in 2008. The annual incidence was $0.21 / 10,000$. Although, the reemergence of malaria in central China was controlled in 2008 , the number of malaria cases and the incidence of the disease in this region still accounts for $68 \%$ of the total cases reported in the country [6].

Although great success has been achieved since the launch of National Malaria Control Programme in 1955, malaria remains a serious public health problem in China [7-9]. Falciparum malaria, the most deadly among the four main types of human malaria, accounted for $14.9 \%$ of all blood-test confirmed malaria cases in 1998 [10]. Falciparum malaria had been endemic in fifteen provinces of China in the early 1950s. The endemic area of falciparum malaria was restricted to eight provinces by 1980 , and only to two provinces, Yunnan and Hainan, by 1998 [10]. Consequently, knowledge regarding the distribution of this disease vector, the genetic characteristics of its populations in relation to local environmental conditions is valuable for malarial control.

An. sinensis belongs to Hyrcanus group that includes approximately 30 morphologically indistinguishable species. In China, 25 taxa, including synonyms, have been recorded [11]. The study on the group in China can be traced back to the 1930s. Yao and Wu [12] and Yao and Ling [13] observed that An. sinensis could be separated into different forms on the basis of the egg deck width. Baisas and $\mathrm{Hu}$ [14] described the form with narrow decked eggs as a new species, An. lesteri. Ho et al. [15] also reported $A n$. lesteri in China, but they noticed some distinct bionomic characters found in China as compared to those of the Philippine form. Particularly, the Chinese form of $A n$. lesteri has a strong preference for human blood [15].

Cytogenetic studies have revealed two karyotypic forms, A (XY1) and B (XY2), in An. sinensis [16], which correspond to distinct sequences of the second internal transcribed spacer (ITS2) region [17]. Both forms were present in Thailand [16], but only form B was observed in China and Korea [17,18]. Analyses of microsatellites loci revealed high polymorphic nature of the An. sinensis populations in China, which indicates that An. sinensis could be divided at least into two populations [19]. Recent molecular studies suggested that $A n$. lesteri from Korea and Japan, An. lesteri from China and An. lesteri from the Philippines were all the same species [20]. Ree et al. [21] reported an unknown Anopheles species that was morphologically identical to An. sinensis, and $\mathrm{Li}$ et al. [22] also observed two unknown species that were morphologically similar to $A n$. sinensis. Rueda [23] designated these two species as new species,
An. belenrae sp. Nov. and An. kleini sp. Nov, based on their same morphologically identity with An. pullus and An. sinensis, respectively. Morphological identification of sinensis complex is extremely difficult, so that some members of $A n$. lesteri, An. pullus (including the form yatsushiroensis) and two species (at least) are mixed in the population of $A n$. sinensis in Korea.

Various levels of population subdivision of the anopheline mosquito species have been observed from nearly panmictic across a wide geographic range to highly divergent within a short distance. A high rate of recurrent gene flow and/or recency of population expansion has made the populations of some anopheline species hardly differentiated [24-26]. However, other species are composed of highly structured populations, due primarily to geographic barriers, such as mountain chains or arid valleys [27-29]. These genetic data are of special value since it may enable planning of effective strategies for malaria control [30]. For instance, the degree of dispersal of insecticide resistant individuals or genetically modified mosquitoes may provide insight for the development of novel mosquito control strategies. Therefore, understanding the genetic structures of vector species may contribute not only to predict the spread of genes of interest (such as insecticide resistant or refractory genes), but also to identify heterogeneities in disease transmission due to distinct vector populations.

A recent microsatellite study revealed two genetic pools indicating the coexistence of two genetic units in the sampled sites [31]. In this study moderate genetic differentiation was identified in the An. sinensis populations in China and the population divergence was not correlated with geographic distance or barrier in the range [19]. A better knowledge of the status of genetic structure in the local An. sinensis populations should benefit malaria control programs in China and enable more appropriate control strategies to be developed. Unfortunately, there is very limited information about An. sinensis population genetics.

Mitochondrial DNA remains one of the most powerful and reliable tools for detecting population structure and inferring population differences due to its high and rapid mutational rate compared with nuclear DNA [32]. Interestingly, it has been found that within mtDNA, there are regions that diverge rapidly, while other regions that are highly conserved, making the different regions suitable for analysis of different taxonomic levels [33,34]. Mitochondrial ND5 gene was previously used as a powerful tool in elucidating the level of genetic and phylogenetic divergence between closely related species [35]. Herein, we provide data that describe/discuss the population structure, genetic variability and gene flow among An. sinensis populations from China based on NADH of dehydrogenase subunit 5 (ND5) gene of mitochondrial DNA (mtDNA). 


\section{Methods}

\section{Mosquito collection and identification}

An. sinensis mosquitoes used in this study were collected during the period of July 2010 to September 2012 by light traps from ten sites located in seven provinces in China (Table 1 and Figure 1). These sites are highly diverse in environmental conditions, malaria incidence, and mosquito composition and density. Central and northern Chinese provinces (e.g. Henan) are characterized by a short summer period (June - August). The southern and south western provinces (e.g. Yunnan and Hainan) are identical to subtropical areas, experiencing relatively high temperature. There is a significant threat of malaria mortality and morbidity in some Chinese provinces such as Yunnan, Hainan and Henan.

An. sinensis, obtained from different locations, were morphologically identified using keys described by Lu et al. [36]. The identification was confirmed by specificspecies PCR based on rDNA ITS2, according to the Ma et al. [37] protocol. Total genomic DNA for individuals used in the study were isolated using extraction kit (QIAGEN, QIAamp DNA Mini Kit) following the manufacturer's instructions.

Primer design, genomic DNA isolation, amplification and sequencing of rDNA ITS2 and ND5 of mtDNA fragments

A forward and reverse primer pair (5'-TGTGAACTG CAGGACACAT-3' and 5'-GTTCTA CGGGCCTATCA CC-3'), corresponding to ITS2 that was invariably conserved in An. sinensis regardless of the region of collection, was synthesized. This primer pair was used to amplify ITS2 fragment from genomic DNA samples followed by sequencing the amplified DNA fragment to verify the collected individual mosquitoes as true An. sinensis. Another forward and reverse primer pair (5'-TTGCGCCTAATCCTGCTAT3 ' and 5'-TGATTTGTGGTGTCAATGT-3') were designed and synthesized for subsequent amplification of ND5 DNA fragments from An. sinensis genomic DNA samples. The
ND5 primers were designed based on highly conserved regions of all available mosquito ND5 sequences from GenBank databases. Primer 3 software program was used to adjust the annealing temperature of two primer pairs. The primer pairs were used to amplify ND5 DNA fragments from genomic DNA samples extracted from $A n$. sinensis collected from different regions to assess the polymorphism of the ND5 gene.

The PCR was conducted in a $50 \mu \mathrm{L}$ reaction mixture containing $1 \mu \mathrm{L}$ of a 1:200 diluted genomic DNA, 25 pmol primers, $5 \mu \mathrm{L}$ 10X reaction buffer, $2.5 \mathrm{mM} \mathrm{MgCl} 2$, $200 \mu \mathrm{M}$ of each $\mathrm{dNTP}$, and $1 \mathrm{U}$ Taq polymerase. The cycling condition was 5 minutes denaturation at $94^{\circ} \mathrm{C}$, followed by 35 cycles of 15 seconds denaturation at $94^{\circ} \mathrm{C}, 15$ seconds annealing at $50^{\circ} \mathrm{C}$, and 1 minute extension at $72^{\circ} \mathrm{C}$, ending with a final extension for $5 \mathrm{~min}$ at $72^{\circ} \mathrm{C}$. Amplified PCR products were purified and then unidirectionally sequenced for both strands for each one of the two regions by Sangon Biotech (Shanghai Co., Ltd., Beijing, China).

\section{Sequences alignment and analysis}

The ND5 gene sequences from $140 \mathrm{An}$. sinensis individuals were aligned by using BioEdit program 5.0.9 [38]. The Haplotype diversity, its variance and nucleotide diversity [39] among populations of An. sinensis from different provinces and/or sites, were estimated using the program DnaSP 4.9 [40]. The Tajima [41] and the Fu and $\mathrm{Li}$ [42] tests were used to examine the hypothesis of selective neutrality of nucleotide substitutions. Tajima's $\mathrm{D}$ test was utilized to examine whether the average number of pair-wise nucleotide differences (K) between sequences was larger or smaller than expected from the observed number of polymorphic sites (S).

Population pairwise net genetic distances based on Slatkin's linearised $F_{S T}$ [43], and hierarchical analysis of molecular variance (AMOVA) [44,45] were estimated Using Arlequin 3.5. 1.3 [46]. The significance of $\mathrm{F}_{S T}$

Table 1 Collection data of An. sinensis populations in China

\begin{tabular}{|c|c|c|c|c|}
\hline Population (code) & Collection site & Collection date & $\mathrm{n}$ & Coordinats \\
\hline Hainan (HA) & Qiongzhong & July-August, 2011 & 09 & $19^{\circ} 05 \mathrm{~N}, 109^{\circ} 83 \mathrm{E}$ \\
\hline Henan (HE-N) & Yongcheng North & Nov., 2010 & 09 & $33^{\circ} 42 \mathrm{~N}, 115^{\circ} 58 \mathrm{E}$ \\
\hline$(\mathrm{HE}-\mathrm{S})$ & Yongcheng South & Oct., 2010 & 19 & $34^{\circ} 18 \mathrm{~N}, 116^{\circ} 39 \mathrm{E}$ \\
\hline Jiangxi (JX-N) & Nanchang & July 2011 & 15 & $28^{\circ} 68 \mathrm{~N}, 115^{\circ} 89 \mathrm{E}$ \\
\hline$(J X-J)$ & Jingdezhen & August 2011 & 12 & $29^{\circ} 3 \mathrm{~N}, 117^{\circ} 22 \mathrm{E}$ \\
\hline Guangxi (GX) & Guilin & July, 2011 & 10 & $25^{\circ} 29 \mathrm{~N}, 110^{\circ} 28 \mathrm{E}$ \\
\hline Shanghai (SH) & Baoshan & August 2011 & 15 & $31^{\circ} 41 \mathrm{~N}, 121^{\circ} 48 \mathrm{E}$ \\
\hline Sichuan (SC-C) & Chengdu & August 2011 & 07 & $30^{\circ} 67 \mathrm{~N}, 104^{\circ} 06 \mathrm{E}$ \\
\hline$(\mathrm{SC}-\mathrm{N})$ & Nanchong & August 2011 & 17 & $33^{\circ} 94 \mathrm{~N}, 106^{\circ} 02 \mathrm{E}$ \\
\hline Yunnan (YN) & Gaoligong Mountain & September 2012 & 27 & $25^{\circ} 11 \mathrm{~N}, 98^{\circ} 40 \mathrm{E}$ \\
\hline
\end{tabular}

$\mathrm{n}=$ sample size. 


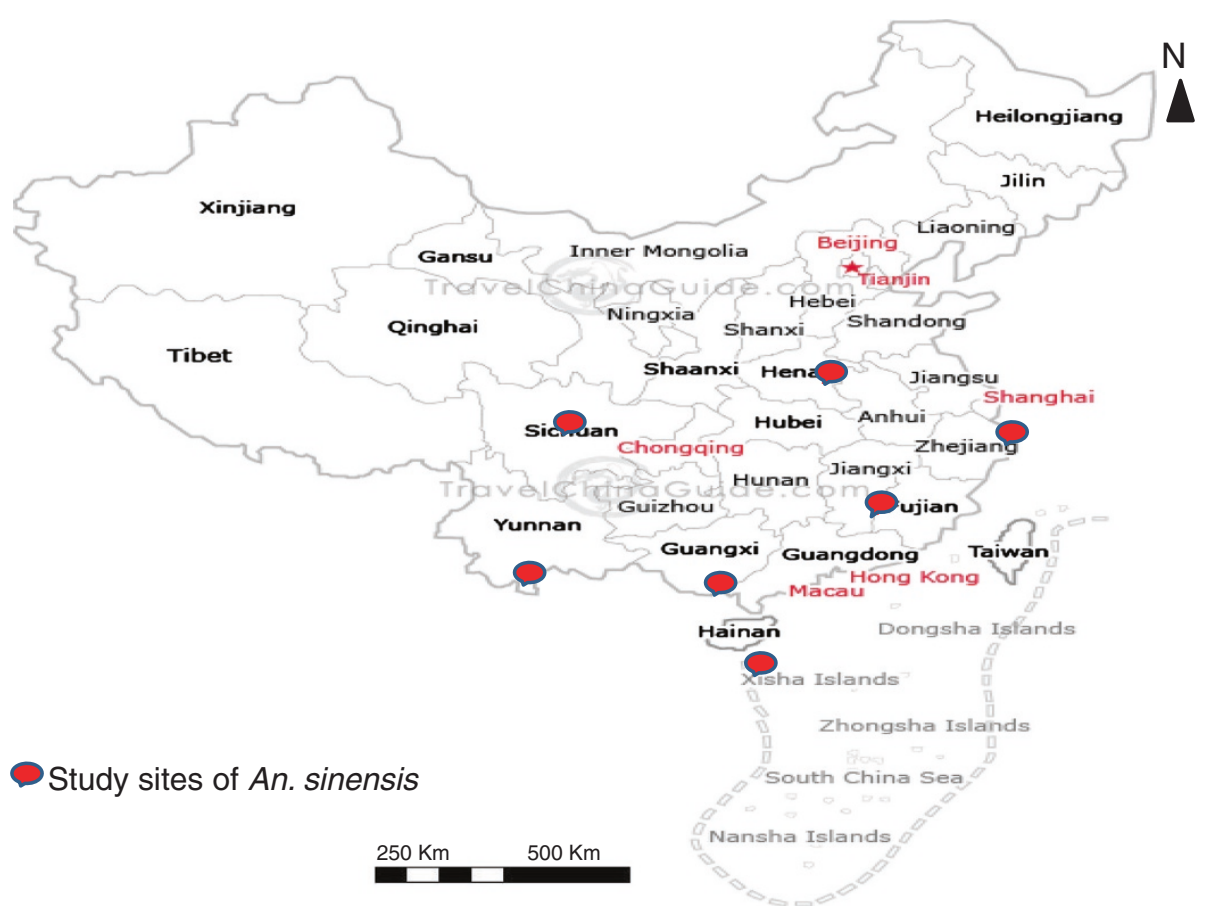

Figure 1 China map shows study sites.

evaluated was based on 1023 random permutations. Demographic analysis and spatial parameters were assessed using the distribution of pair wise sequence differences (mismatch distribution) of Rogers and Harpending [47]. We used Tajima's D [41] and Fu's Fs statistics [48]. The significance of the $D$ and $F$ s values of Tajima and $\mathrm{Fu}$, respectively, was evaluated by comparison with randomly generated values based on the observed (S) with 10,000 repeats. The Mantel test [49] was performed to test significant correlation between population genetic distance and linear straight geographical distances.

A TCS software program $2.1[50,51]$ was used to construct a haplotypes network and estimated genealogy relationship between haplotypes of An. sinensis populations collected from different sites in China.

\section{Ethical approval}

We obtained ethical approval from the Ethical Review Committee of Chinese Center for Disease Control and Prevention (No.201214). Permission was also obtained from the directors and related departments of provincial $\mathrm{CDC}$ in seven provinces in China.

\section{Results}

\section{An. sinensis identification}

All specimens used in this study were confirmed as $A n$. sinensis either by PCR based on species-specific ITS 2 sequences. Alignment of 30 representative ITS2 sequences of rDNA (accession number KC769646), in conjunction with different sites of collection, confirmed that only sequences of $A n$. sinensis were used.

\section{Characterization and Haplotypes estimation of ND5}

A 836-bp segment of mitochondrial DNA (mtDNA), corresponding to coding region of $\mathrm{NADH}$ dehydrogenase gene subunit 5 (ND5), was successfully amplified. Analyses were carried out on 729 bp of mtDNA dehydrogenase gene subunit 5 (ND5) from $140 \mathrm{An}$. sinensis individuals collected from ten sites in seven Chinese Provinces. A total of 84 haplotypes of mtND5 were determined in An. sinensis individuals collected from China (GenBank accession numbers: KC565754 - KC565837), in which 71 polymorphic sites were detected within these populations. The partial nucleotide sequences of ND5 gene showed variable polymorphic sites within and among An. sinensis populations. The frequencies of haplotypes clearly divided these populations into three clusters. Among them, one cluster $(n=51)$ had low variable sites including ancestral populations, another cluster $(n=32)$ displayed moderate polymorphism (at least two main constant variable sites) with nucleotide substitutions at positions (55 and 325) respectively, and the third population $(n=57)$ showed many variable sites with one main constant site at position 529. No characteristics of heteroplasmy and insertion or deletion events were shown within the analysed mtDNA sequence.

The other nucleotide substitutions in ND5 gene of $A n$. sinensis populations were synonymous (Additional file 1: 
Table S1). There were seven informative non-synonymous substituted sites with amino acids replacement. The first substitution site that corresponding to position 258 (N258S) only found in Yunnan population. Replacement corresponding to position 283 (K283N) occurred in seven populations (GX, HA, HE-N, HE-S, JX-J, JX-N and SH). Substitution at position 309 (N309S) was found in populations (JX-J, HE-S and SH). Substitutions at positions 379 and 385 (R379S; K385N), respectively, were detected in seven populations (HA, HE-S, JX-J, JX-N, SC-N, SH and YN). Substitution at position 529 (K529N) occurred in all populations except GX. Also substitution at position 687 (I687T) was detected in all sites except YN (Additional file 1: Table S1).

\section{Genealogical relationships among populations groups of An. sinensis}

The haplotypes (Additional file 1: Table S1) and genealogy network (Figure 2) revealed a network with three clusters separated by a single nucleotide differences. Each one of these networks represents group of haplotypes clustered together with common haplotype and all connected together with ancestral group. In addition to identifying unique observable haplotype frequency, the network analysis also detected the expected mutations and mutational steps that separated between haplotypes. These networks clearly illustrated the genealogical relationship between haplotypes and therefore, the illustration proposed subdivision of $A n$. sinensis populations into three clusters.

\section{Genetic differentiation of $A n$. sinensis populations}

Table 2 shows the analyzed results of $A n$. sinensis populations. Thus, haplotype diversities were ranged from 0.7 to 1.00; average number of nucleotide differences was $2.0-$ 9.19, nucleotide diversities; and average number of mutations was $0.0027-0.013$ and $0.002-0.02$, respectively. The pairwise differences within the populations of $A n$. sinensis were found in the range of $F_{S T}=0.0007-0.341$ and gene flow of $\mathrm{Nm}=0.97-769.5$ with obviously significant difference in Yunnan province as compared to other sites (Table 3).

Tajima's D test of departures from the neutral expectations did not show significant deviation from neutrality. However, $\mathrm{Fu}$ and Li's $D$ and $F$ statistics indicated a significant deviation from neutrality only for overall

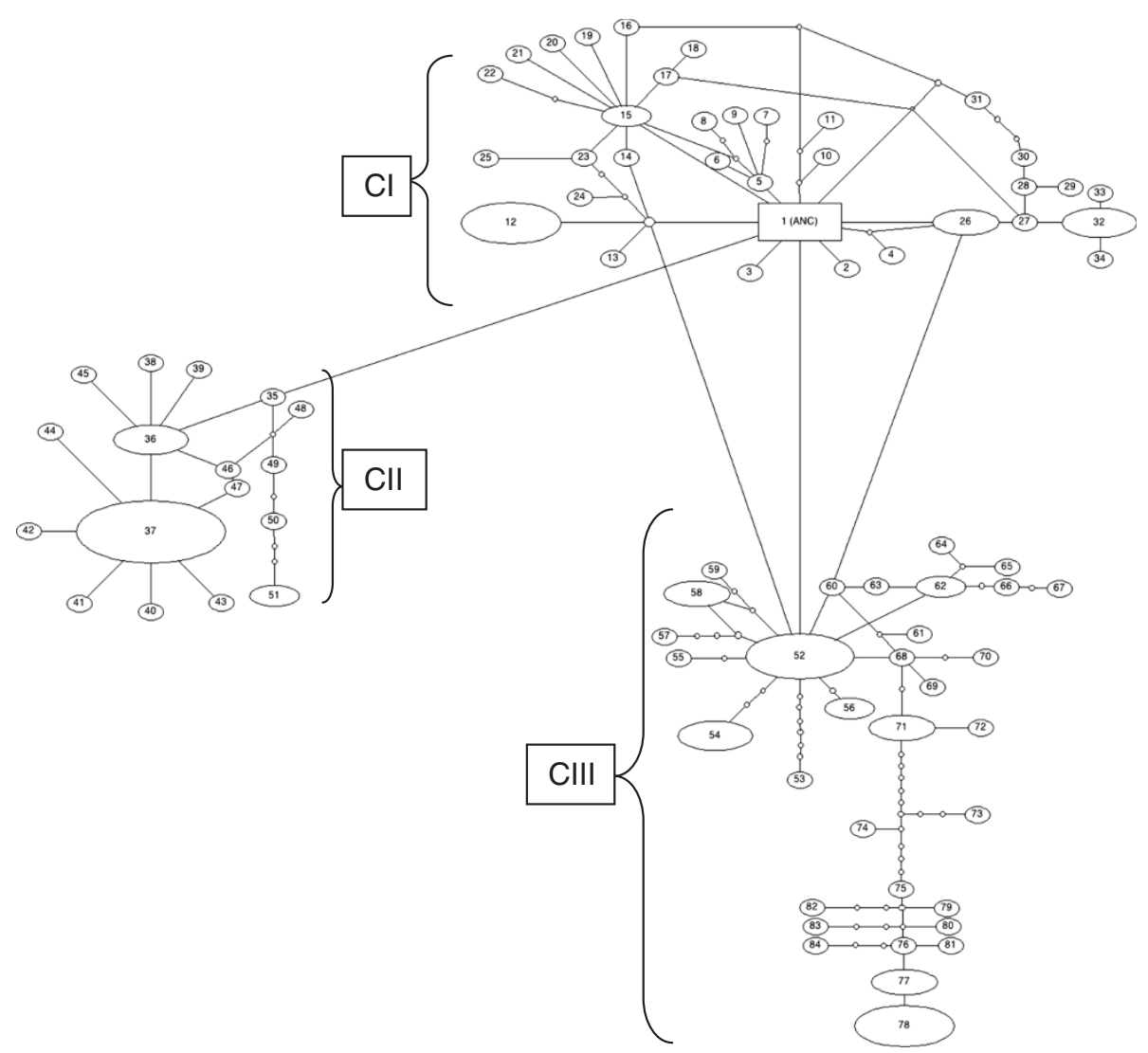

Figure 2 Haplotypes network of An. sinensis populations collected from China based on 729bp of mtDNA ND5 gene. The clusters from each other are differentiated by a single mutation step. The size of each observed ellipse indicates the proportion of the haplotype in populations, the ancestral (ANC) haplotype is rectangular. Small circle is an expected mutation. $C=$ cluster. 
Table 2 Haplotypes and nucleotide diversity of An. sinensis populations collected from China

\begin{tabular}{lcccccc}
\hline Population & S & H & Hd \pm SD & K & $\boldsymbol{\pi} \pm$ SD & $\boldsymbol{\theta}$ \\
\hline HA & 28 & 9 & $1.00 \pm 0.05$ & 6.19 & $0.0128 \pm 0.003$ & 0.015 \\
HE-N & 13 & 9 & $1.00 \pm 0.05$ & 3.83 & $0.0053 \pm 0.0007$ & 0.007 \\
HE-S & 35 & 17 & $0.988 \pm 0.02$ & 6.56 & $0.009 \pm 0.002$ & 0.014 \\
JX-J & 27 & 12 & $1.00 \pm 0.03$ & 6.53 & $0.009 \pm 0.002$ & 0.014 \\
JX-N & 25 & 15 & $1.00 \pm 0.02$ & 6.19 & $0.0086 \pm 002$ & 0.011 \\
GX & 13 & 8 & $0.93 \pm 0.08$ & 3.64 & $0.005 \pm 0.001$ & 0.006 \\
SC-C & 4 & 3 & $0.714 \pm 0.13$ & 2.00 & $0.0027 \pm 0.0005$ & 0.002 \\
SC-N & 26 & 11 & $0.882 \pm 0.07$ & 5.32 & $0.0073 \pm 0.002$ & 0.011 \\
SH & 29 & 14 & $0.991 \pm 0.03$ & 7.44 & $0.010 \pm 0.002$ & 0.013 \\
YN & 25 & 8 & $0.849 \pm 0.04$ & 9.16 & $0.013 \pm 0.001$ & 0.009 \\
Overall** & 71 & 84 & $0.981 \pm 0.004$ & 7.17 & $0.0098 \pm 0.0007$ & 0.02
\end{tabular}

Tajima's D = Not significant for all, Fu \& Li's D \& F were not significant for all except for overall**. $\mathrm{S}=$ number of segregating sites, $\mathrm{H}=$ number of haplotypes, $\mathrm{K}=$ Average number of nucleotide differences, $\mathrm{Hd}=$ Haplotypes diversity, $\pi=$ Nucleotide diversity, SD = standard deviation, $\theta=$ Average number of mutation per sequence. For population abbreviations see Table 1.

populations $(P<0.01)$, suggesting the existence of excessive rare nucleotide polymorphisms, with possible effects of purifying, selection, or population expansion. For other populations, all statistics tests did not reveal significant differences, suggesting that the nucleotide substitutions of the ND5 gene are consistent with neutral evolution theory.

\section{Population structure of clustered groups of An. sinensis populations}

Based on the haplotype polymorphism data of the mtDNA, the results revealed the presence of three clustered populations of An. sinensis in China (Additional file 1: Table S1), with similar findings supported by haplotype network (Figure 2). These results indicated the pattern of population structure within An. sinensis in
China. AMOVA results shown in Table 4 revealed that most of the genetic variance lay within the populations $(63.57$ - 72.51\%) than among the populations (27.49 $36.43 \%)$. Three clustered populations had similar results, with a high proportion of the overall genetic variation being attributed within population levels (Table 4). On average, a relatively high percentage of the total genetic variation was attributable within cluster comparisons for all samples (68.68\%). The same finding was confirmed by a significant genetic differentiation in comparisons at the hierarchical level among populations within clusters $\left(F_{S T}=0.364,0.275,0.332\right.$ and $\left.0.313 ; P<0.0000\right)$ in the three clusters or overall populations respectively.

\section{Demographic and spatial history of An. sinensis populations in China}

The distribution of pairwise nucleotide differences for the total An. sinensis samples, revealed generally patterns, characteristic of a population that has undergone a large expansion. According to the mismatch distribution analysis, the hypothesis of spatial experienced expansion could not be rejected in the three clusters and overall samples. A good fit of the spatial expansion was also observed for all clustered samples based on Sum of Squared deviation (SSD) values that were not significant in all the cases (Table 5). With these results, our data support population growth and range expansion of $A n$. sinensis. We also calculated the sum of squared deviation and raggedness index under the demographic expansion model for each cluster and found that all populations in the three clusters had low and nonsignificant values (Table 5).

Neutrality tests of Tajima's $D$ revealed non-significant negative values in all individual populations of the three clusters and pooled samples (Table 5). However, negative, large and highly significant Fu's $F$ s values were observed $(P<0.001)$ in the three clustered populations and

Table 3 Genetic differentiation and gene flow between populations of An. sinensis collected from China

\begin{tabular}{|c|c|c|c|c|c|c|c|c|c|c|}
\hline Population & $\mathrm{HA}$ & HE-N & HE-S & $J X-J$ & $J X-N$ & GX & SC-C & SC-N & $\mathrm{SH}$ & YN \\
\hline $\mathrm{HA}$ & & 3.75 & 42.43 & 13.76 & -25.35 & 2.22 & 2.04 & 5.34 & 23.1 & 42.33 \\
\hline HE-N & 0.118 & - & 9.61 & 769.5 & 27.3 & 6.12 & 5.03 & 4.6 & 26.83 & 1.53 \\
\hline HE-S & 0.012 & 0.05 & - & -78.93 & -47.46 & 2.05 & 1.75 & 3.25 & 55.64 & 3.93 \\
\hline$J X-J$ & 0.035 & 0.0007 & 0.006 & - & -18.54 & 3.16 & 2.81 & 4.33 & 22.32 & 3.1 \\
\hline$J X-N$ & -0.020 & 0.018 & 0.011 & -0.028 & - & 3.17 & 2.87 & 6.27 & -29.24 & 4.9 \\
\hline GX & 0.184 & 0.076 & 0.196 & 0.137 & 0.136 & - & -10.34 & -49.76 & 4.79 & 1.12 \\
\hline SC-C & 0.197 & 0.090 & 0.222 & 0.151 & 0.149 & -0.051 & - & -165.83 & 3.16 & 0.97 \\
\hline SC-N & 0.085 & 0.098 & 0.133 & 0.104 & 0.074 & -0.010 & -0.003 & - & 7.91 & 1.95 \\
\hline $\mathrm{SH}$ & 0.021 & 0.018 & -0.009 & -0.023 & -0.017 & 0.095 & 0.137 & 0.06 & - & 4.74 \\
\hline YN & $0.011^{*}$ & $0.246^{* *}$ & $0.113^{* *}$ & $0.139 * *$ & $0.093^{* *}$ & $0.309^{* *}$ & $0.341^{* * *}$ & $0.204^{* *}$ & $0.095^{* *}$ & - \\
\hline
\end{tabular}

Significance of $X^{2}:{ }^{*} P<0.05, * * P<0.01,{ }^{* * *} P<0.001 . F_{S T}=$ genetic differentiation between populations, $N m=$ gene flow. Numbers above diagonal are $\mathrm{Nm}$, numbers below diagonal are $F_{S T}$. For population abbreviations see Table 1. 
Table 4 AMOVA results among three clustered An. sinensis populations collected from China

\begin{tabular}{|c|c|c|c|c|c|c|}
\hline Cluster & $\begin{array}{l}\text { Source of } \\
\text { variation }\end{array}$ & d. $f$ & $\begin{array}{c}\text { Variance } \\
\text { components }\end{array}$ & $\begin{array}{l}\text { Variation } \\
\text { (\%) }\end{array}$ & $\begin{array}{c}\text { Fixation } \\
\text { Index }\left(F_{\mathrm{ST}}\right)\end{array}$ & P-value \\
\hline \multirow[t]{2}{*}{ | \& ||: } & Among populations & 1 & 0.88 & 36.43 & & \\
\hline & Within populations & 81 & 1.54 & 63.57 & 0.364 & 0.0000 \\
\hline \multirow[t]{2}{*}{ | \& |||: } & Among populations & 1 & 1.21 & 27.49 & & \\
\hline & Within populations & 106 & 3.19 & 72.51 & 0.275 & 0.0000 \\
\hline \multirow[t]{2}{*}{ \| \& II: } & Among populations & 1 & 1.66 & 33.16 & & \\
\hline & Within populations & 87 & 3.34 & 66.84 & $0 . .332$ & 0.0000 \\
\hline \multirow[t]{2}{*}{ Overall: } & Among populations & 2 & 1.26 & 31.32 & & \\
\hline & Within populations & 137 & 2.76 & 68.68 & 0.313 & 0.0000 \\
\hline
\end{tabular}

d.f. $=$ degree of freedom. Significance tests (1023 permutations).

pooled samples (Table 5). Fu's $F$ s is considered to be more sensitive in detecting deviations from neutrality and thereby suggesting possible population expansion. The expansion may have been restricted to separate local areas that resulted in the non-significant negative Tajima's $D$ values for clusters and all populations. The Mantel test results showed in Figure 3 supported the AMOVA results, that entire pooled populations lack of correlations between genetic $\left(F_{S T}\right)$ and geographic distance $\left(\mathrm{R}^{2}=0.058, P=0.301\right)$.

\section{Abundance of An. sinensis population groups in different Chinese provinces}

Although all three clusters were observed in An. sinensis samples collected from different sites, there were apparent differences in relative abundance for given groups. Figure 4 illustrates the distribution of the three clustered populations of $A n$. sinensis in different geographical locations in China. Hainan and Yunnan provinces showed high abundance of cluster III (78\% and 74, respectively). In contrast, CII became the majority in Guangxi and Sichuan (70\% and 58\%, respectively). Although CI was present in all sites, it was noticeably more abundant than the other two clusters in Henan and Jiangxi (47\% and $56 \%$, respectively). Shanghai showed relatively even distribution of the three populations (Figure 4).

\section{Discussion}

It has generally been accepted that the degree of the polymorphisms of ND5 gene serves as a dependable representative for assessing the genetic variations in populations of a given species [35]. The present study described an analysis of 729 bp sequence of ND5 mitochondrial DNA in An. sinensis populations obtained from 10 collection sites in seven Chinese provinces. The results indicate that $A n$. sinensis populations in China were highly polymorphic in the ND5 gene. In most populations high level of polymorphism and moderate genetic differentiations were detected in this mosquito $\left(F_{S T}=0.001-0.341\right)$. These results are comparable to previous findings on $A n$. sinensis and its member group of Hyrcanus $A n$. lesteri $[19,52,53]$. Also these results are in agreement with $A n$. baimaii in India [54], An. darlingi malaria vector in Central and South America [55-57], An. albimanus [58] in Latin America and African malaria vectors An. gambiae [24], An. arabiensis and An. funestus $[44,59,60]$.

Neutrality tests of Tajima's $D, \mathrm{Fu}$ and Li's $D$ and $F$ did not detect deviation from neutrality theory in the ND5 gene within any population of $A n$. sinensis. Only Fu and Li's $D$ and $F$ tests were significant when all populations from different sites pooled together, and this perhaps is due to a high level of polymorphism within ND5 gene $(71 / 140)$. These results support the neutral theory of

Table 5 Mismatch and neutrality tests results of three clustered An. sinensis populations collected from China

\begin{tabular}{|c|c|c|c|c|c|c|c|c|}
\hline \multirow[b]{3}{*}{ Cluster } & \multirow[b]{3}{*}{$\mathbf{N}$} & \multirow[b]{3}{*}{$S$} & \multicolumn{4}{|c|}{ Mismatch } & \multicolumn{2}{|c|}{ Neutrality Tests } \\
\hline & & & \multicolumn{2}{|c|}{ Demographic } & \multicolumn{2}{|c|}{ Spatial } & \multirow{2}{*}{$\begin{array}{c}\text { Tajima's } \\
D\end{array}$} & \multirow{2}{*}{$\begin{array}{c}\text { Fu's } \\
\text { Fs }\end{array}$} \\
\hline & & & SSD & Rag & SSD & Rag & & \\
\hline I & 51 & 27 & $0.005(0.11)$ & $0.033(0.17)$ & $0.005(0.16)$ & $0.033(0.17)$ & -1.3884 & $-26.1251^{* * * *}$ \\
\hline$\|$ & 32 & 17 & $0.010(0.46)$ & $0.049(0.56)$ & $0.010(0.33)$ & $0.049(0.56)$ & -1.2669 & $-11.0673^{* * * *}$ \\
\hline III & 57 & 50 & $0.016(0.29)$ & $0.009(0.72)$ & $0.016(0.2)$ & $0.009(0.72)$ & -0.5710 & $-11.3590^{* *}$ \\
\hline Overall & 140 & 84 & $0.011(0.29)$ & $0.031(0.23)$ & $0.011(0.23)$ & $0.031(0.48)$ & -1.0750 & $-16.1840^{* *}$ \\
\hline
\end{tabular}

$\mathrm{N}=$ sample size, $\mathrm{S}=$ Segregating sites, $\mathrm{SSD}=$ Sum of Squared deviation, Rag $=$ Harpending's Raggedness index. Significance ** $P<0.001, * * * * \mathrm{P}<0.0000$, all $=$ all samples together (Un-clustered). No Tajima's D P-values are significant. Number in parentheses is $\mathrm{P}$ value. 


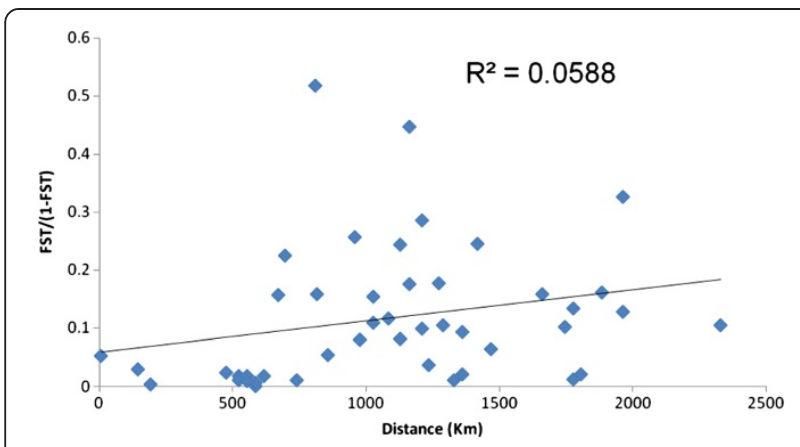

Figure 3 Correlation between average $F_{S T}$ and geographic distance between collection sites for pairwise comparisons of An. sinensis populations. $P=0.301$

mitochondrial gene evolution and suggested that there is a large effective population size of An. sinensis in these locations.

Generally, the results showed that there is low-to-moderate genetic differentiation and high gene flow within different populations except the $\mathrm{YN}$ population that displayed a significantly high level of genetic differentiation and restricted gene flow with all populations except Hainan population $\left(F_{S T}=0.011 ; N m=42.33\right)$. This could be due to bias of low sample size in Hainan $(n=9)$ but similar samples were generated from (HE-N and SC-N), that give significant results with YN. Moreover, the two sites of Hainan and Yunnan are somehow similar in their conditions for mosquito habitats. Although distantly apart, both sites belong to typical temperate tropic zones where there are great seasonal variations in abundance of mosquito populations that reach their peak during summer [61]. Yunnan is noted as centre of biodiversity [61-64]; because of it's a highly complex region topographically due to its transitional position from tropical southern Himalayas to eastern Asia and from tropical Southeast Asia to subtropical China as well as at the junction of the India and Burmese plates, derived from Gondwanaland, and the Eurasian plate [65]. Further studies in these areas could be established by collecting and analysing more $A n$. sinensis samples over several seasons. The climatic condition could impose selection on $A n$. sinensis populations. In addition, $A n$. sinensis breeds in a wide range of habitats including natural and artificial [4,64].

Interestingly, the results of haplotypes and genealogy revealed the presence of three clustered populations of An. sinensis across collection sites in China. In general cluster I approximately represented $36 \%(\mathrm{n}=51)$, cluster II includes relatively less samples $23 \%(n=32)$ where $41 \%$ ( $\mathrm{n}=57$ ) belong to cluster III. Cluster I was found in all populations collected across the study zone (ancestral), cluster
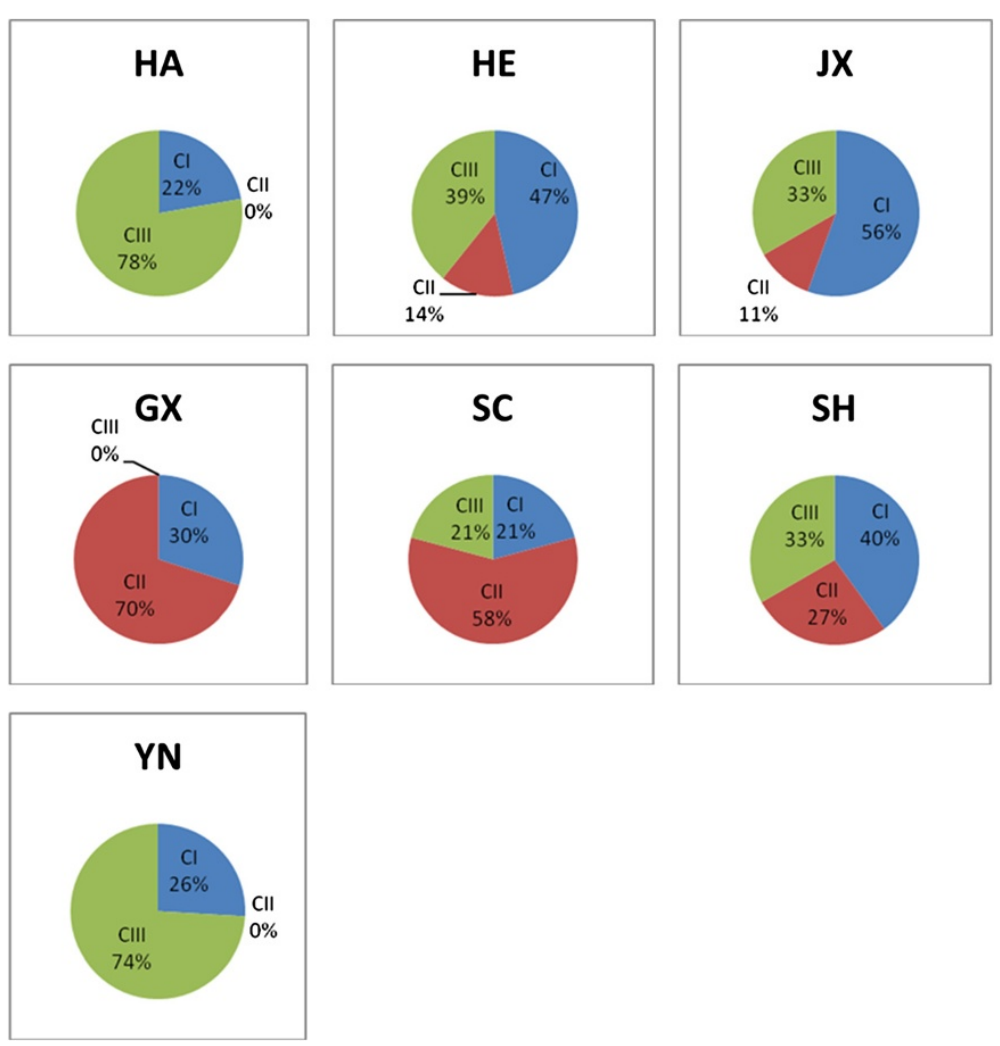

Figure 4 Abundance of An. sinensis population clusters in Chinese provinces. 
two was found in 8 populations out of 10 (except HA and $\mathrm{YN}$ ) where cluster III occured in 9 populations (except SCC). The three clustered populations were assigned by at least one common haplotype or nucleotide substitution and/or amino acid(s) replacement. The proportion of the three clustered populations varied from site to site in China. For example, the cluster three of An. sinensis population is abundant in Hainan and Yunnan, but moderately abundant in Shanghai and Henan and absent in Guangxi. These results are similar to those reported in a previous study [19] that indicated that there were two gene pools found within An. sinensis populations collected across China from 20 collection sites (one pool included six populations and the second pool included eight populations). In that study the author reported that there was coexistence between two gene pools and specimens were assigned to one gene pool when it is remaining greater than $80 \%$. A similar finding was reported in South Korea that An. sinensis includes two groups associated with the presence of a mountain functioning as a genetic barrier [52]. Normally once there are two populations possible, there is, an intermediate group. In present study cluster II could be an intermediate group. In this cluster (C II) obviously only a few individuals share polymorphic sites with each one of the other two clusters (Additional file 1: Table S1), which could be due to an ancestral background.

An AMOVA analysis using the three clusters found that $31.32 \%$ of the variance was attributed to between populations and $68.68 \%$ to within populations. A similar phenomenon of variance pattern was observed in comparing three clusters. There is substantial significant genetic differentiation among populations of the three clusters $\left(F_{S T}=0.313 ; P<0.0001\right)$. The level of genetic differentiation is relatively similar to that previously detected in $A n$. sinensis populations in China using microsatellites [19]. The distribution of the three clusters does not seem to be attributable to geographical range because no correlation between genetic and geographic distance was detected based on the mantel test results. Moreover, sympatric occurrence of more than one clustered populations in the same study site is further evidence that distance has no major impact on genetic differentiation. Similar results have been reported for An. gambiae and An. arabiensis malaria vectors in Africa [66]. In that report, the authors suggested that the high level of differences in these populations was likely due to some reproductive isolations rather than physical barrier or distance and also possibly due to demographic history or ecological diversification. The wide range of distributions for this mosquito population of An. sinensis is an importance factor for malaria control and that suggests further examining at a local level for microgeographic scales in lack of isolation by distance (IBD), for understanding population connectivity and vector dispersal across multiple spatial scales.
Mismatch distribution of demographic and spatial analyses were assessed using the distribution of pairwise sequence differences and suggested population expansion of An. sinensis in China. Both SSD and raggedness index supported the evidence of population expansion. Moreover, negative and significant values of Fu's $F s$ are further indicators for population growth. These findings are also consistent with pervious reported results of An. sinensis in South Korea where two groups of $A n$. sinensis have recently experienced expansion in population size based on mitochondrial control region [52].

Genetic diversity is a key factor that enables adaptation and persistence of a natural population towards changing or adverse environmental conditions. Its remains true that our analyses are based on a single marker, mtDNA, which may not be representative of the genome as a whole. Although most of the polymorphism in mtDNA sequences is likely to be neutral, positive or negative selection on any site within the mtDNA genome will influence the pattern of variation across the whole molecule [67]. Our results emphasize the need for further investigation with deeper sampling (especially in the areas where there are more than one cluster) using more polymorphic nuclear markers to elucidate the forces that shape and maintain the population structure. Further studies are required to investigate the three clusters of An. sinensis regarding ecology and susceptibility to malaria transmission.

\section{Conclusion}

These results have implication for the interpretation of genetic population structure in An. sinensis. Our results revealed that the populations of An. sinensis in China are highly polymorphic within the ND5 gene of mitochondrial DNA with moderate genetic variability. There are at least three clustered populations with different abundance within Chinese provinces. The occurrence of more than one sympatric population could be due to adaption of the local environmental factors such as type of breeding sites and its availability in different seasons and/or as well as for control measures. Geographic range does not seem to have a major effect on the genetic variability of $A n$. sinensis. Further exploring the mechanism for the absence of a given population may provide insight towards development of new vector control strategies.

\section{Additional file}

Additional file 1: Table S1. Polymorphic positions of ND5 (mtDNA) of An. sinensis collected from China.

\section{Competing interests}

The authors declare that they have no competing interests. 


\section{Authors' contributions}

AMM performed lab experiments, data interpreting and wrote the manuscript. XBL and SRY conducted the field work and samples collection. QYL conceived and designed the research, supervised the field, lab work and wrote the manuscript. All authors read and approved the final version of the manuscript.

\section{Acknowledgements}

We are grateful to Dr. Fengxia Meng, Dr. Yuhong Guo, Dr. Haixia Wu and Dr. Guichang Li from the Department of Vector Biology and Control, ICDC, China CDC for their support to the mosquito samples. We thank Dr. Jianyong Li from Virginia Tech for the revision of the manuscript. Also we thank Prof. Dia-Eldin A. El naiem from Department of Natural Sciences, University of Maryland Eastern Shore for extensive English and grammatical editing. This study was funded by the National Basic Research Program of China (973 Program) (Grant No.2012CB955500, 2012CB955504) and supported by Chinese Center for Disease Control and Prevention (China CDC). Most of the mosquito samples were collected through the National Selected Disease Vectors Surveillance Networks, China CDC. This study was also funded by the Postdoctoral Program for African Researchers of

China-Africa Science and Technology Partnership Program

(CASTEP; No. ZF2012021)

\section{Author details}

'Department of Vector Biology and Control, State Key Laboratory for Infectious Disease Prevention and Control, National Institute for Communicable Disease Control and Prevention, Chinese Center for Disease Control and Prevention, Beijing 102206, China. ${ }^{2}$ WHO Collaborating Centre for Vector Surveillance and Management, Beijing 102206, China. ${ }^{3}$ China CDC Key Laboratory of Surveillance and Early-Warning on Infectious Disease, Chinese Center for Disease Control and Prevention, Beijing 102206, China. ${ }^{4}$ Department of Biotechnology, College of Applied and Industrial Sciences, University of Bahri, Khartoum, Sudan. ${ }^{5}$ Collaborative Innovation Center for Diagnosis and Treatment of Infectious Diseases, Hangzhou, China.

Received: 12 February 2013 Accepted: 24 September 2013 Published: 8 October 2013

\section{References}

1. Ma SF: Studies on the Anopheles (A) sinensis group of mosquitoes in China, including four new sibling species. Sinozoologia 1981, 1:59-74

2. Tan WL, Wang ZM, Li CX, Chu HL, Xu Y, Dong YD, Wang Z, Chen DY, Liu H, Liu DP, Liu N, Sun J, Zhao T: First report on co-occurrence knockdown resistance mutations and susceptibility to beta-cypermethrin in Anopheles sinensis from Jiangsu Province, China. PLOS ONE 2012, 7:1371-1380.

3. Han ET, Lee DH, Park KD, Seok WS, Kim YS, Tsuboi T, Shin EH, Chai JY: Re-emerging vivax malaria: changing patterns of annual incidence and control programs in the republic of Korea. Korean J Parasitol 2006, 44:285-294.

4. Ree H: Studies on Anopheles sinensis, the vector species of vivax malaria in Korea. Korean J Parasitol 2005, 43:75-92.

5. Zhou SS, Huang F, Wang JJ, Zhang SS, Su YP, Tang LH: Geographical, meteorological and vectorial factors related to malaria re-emergence in Huang-Huai River of central China. Malar J 2010, 9:337.

6. Zhou SS, Wang Y, Fang W, Tang LH: Malaria situation in the People's Republic of China in 2008. Chinese Journal of Parasitology and Parasitic Diseases 2009, 27:45-455.

7. Tang LH: Progress in malaria control in China. Chin Med J 2000, 113:89-92.

8. Chen B, Harbach RE, Butlin RK: Molecular and morphological studies on the Anopheles minimus group of mosquitoes in southern China: taxonomic review, distribution and malaria vector status. Med Vet Entomol 2002, 16:253-265.

9. Zhou SS, Wang Y, Tang LH: Malaria situation in the People's Republic of China in 2005. Zhongguo Ji Sheng Chong Xue Yu Ji Sheng Chong Bing Za Zhi 2006, 24(6):401-403.

10. Tang LH: Research achievement in malaria control in China. Chinese Journal of Parasitology and Parasitic Diseases 1999, 17:257-259.

11. Ma YJ, Yang P, Xu JN, Chen Z, Pan B: Identification of Anopheles lesteri in China (Diptera: Culicidae): morphologic characters, chromosome karyotype and molecular markers. Entomotaxonomia 2005, 27:199-208
12. Yao YT, Wu CC: One year's observation of Anopheles hyrcanus var. sinensis in Nanking. Trans Far- East Assoc Trop Med 1934, 3:2-26. 9th Congr.

13. Yao YT, Ling LC: Notes on the comparative morphological studies of the three types of eggs laid by Anopheles hyrcanus var. sinensis in Nanking. Trans Far-East Assoc Trop Med 1938, 2:831-837. 2

14. Baisas FE, Hu SMK: Anopheles hyrcanus var. sinensis of the Philippines and certain parts of China, with some comments on Anopheles hyrcanus var. nigerrimus of the Philippines. Mon Bull Bur Health Philipp 1936, 16:205-242.

15. Ho C, Chou TC, Chen TH, Hsueh A: The Anopheles hyrcanus group and its relation to malaria in East China. Chin Med J 1962, 81:71-78.

16. Baimai V, Rattanarithikul R, Kijchalao U: Metaphase karyotypes of Anopheles of Thailand and Southeast Asia: The Hyrcanus group. J Am Mosa Control Assoc 1993, 9:59-67.

17. Min GS, Choochote W, Jitpakdi A, Kim SJ, Kim W, et al: Intraspecific hybridization of Anopheles sinensis (Diptera: Culicidae) strains from Thailand and Korea. Mol Cell 2002, 14:198-204.

18. Xu SB, Qu YJ: Studies on chromosomes of thirteen species of anopheline mosquitoes in China. J Med Coll PLA 1991, 6:286-291.

19. Ma $Y$, Yang $M$, Fan $Y, W u$ J, Ma $Y$, et al: Population structure of the malaria vector Anopheles sinensis (Diptera: Culicidae) in China: two gene pools inferred by microsatellites. PLOS ONE 2011, 6:1371-1380.

20. Wilkerson RC, Li C, Rueda LM, Kim HC, Klein TA, Song GH, Strickman D: Molecular confirmation of Anopheles (Anopheles) lesteri from the Republic of South Korea and its genetic identity with An. (Ano.) anthropophagus from China (Diptera: Culicidae). Zootaxa 2003, 378:1-14.

21. Ree HI, Yong TS, Hwang UW: Identification of four species of the Anopheles hyrcanus complex (Diptera: Culicidae) found in Korea using species-specific primers for Polymerase Chain Reaction Assay. Med Entomol Zool 2005, 56:201-205.

22. Li C, Lee JS, Groebner JL, Kim HC, Klein TA, O'Guinn ML, Wilkerson RC A newly recognized species in the Anopheles Hyrcanus complex and molecular identification of related species from the Republic of South Korea (Diptera: Culicidae). Zootaxa 2005, 939:1-8.

23. Rueda LM: Two new species of Anopheles (Anopheles) Hyrcanus group (Diptera: Culicidae) from the Republic of South Korea. Zootaxa 2005, 941:1-26.

24. Lehmann T, Besansky NJ, Hawley WA, Fahey TG, Kamau L, Collins FH: Microgeographic structure of Anopheles gambiae in western Kenya based on mtDNA and microsatellite loci. Mol Ecol 1997, 6:243-253.

25. Donnelly MJ, Twonson H: Evidence for extensive genetic differentiation among populations of the malaria vector Anopheles arabiensis eastern Africa. Insect Mol Biol 2000, 9:357-367.

26. Simard F, Lehmann T, Lemasson JJ, Diatta M, Fontenille D: Persistence of Anopheles arabiensis during the severe dry season conditions in Senegal: an indirect approach using microsatellite loci. Insect Mol Biol 2000 9:467-479

27. Rongnoparut P, Sirichotpakorn N, Rattanarithiku R, Yaicharoen S, Linthicum K : Estimates of gene flow among Anopheles maculatus populations in Thailand using microsatellite analysis. Am J Trop Med Hyg 1999, 60:508-515.

28. Lehmann T, Hawley WA, Grebert H, Danga M, Atieli F, Collins FH: The Rift Valley complex as a barrier to gene flow for Anopheles gambiae in Kenya. J Hered 1999, 90:613-621.

29. Scarpassa VM, Tadei WP, Suarez MF: Population structure and genetic divergence in Anopheles nuneztovari (Diptera: Culicidae) from Brazil and Colombia. Am J Trop Med Hyg 1999, 60:1010-1018.

30. Collins FH, Besansky NJ: Vector biology and control of malaria in Africa. Science 1994, 264:1874-1875.

31. Collins FH, Paskewitz S: A review of the use of ribosomal DNA (rDNA) to differentiate among cryptic Anopheles species. Insect Mol Biol 1996, 5:1-9.

32. Zink RM, Borrowclough GF: Mitochondrial DNA under siege in avian phylogeoraphy. Mol Ecol 2008, 17:2107-2121.

33. Simon C, Frati F, Beckenbach A, Crespi B, Liu H, Flook P: Evolution, weighting, and phylogenetic utility of mitochondrial gene sequences and a compilation of conserved polymerase chain reaction primers. Ann Ento Soc Am 1994, 87:651-701.

34. Meyer $\mathrm{A}$ : Shortcomings of cytochrome $b$ gene as a molecular marker. Tre Ecol \& Evol 1994, 9:278-280

35. Krzywinski J, Wilkerson RC, Besansky NJ: Evolution of mitochondrial and ribosomal gene sequences in anophelinae (Diptera: Culicidae): implications for phylogeny reconstruction. Mol Phyl Evol 2001 18(3):479-487. 
36. Lu BL: Fauna Sinica, Insect Diptera: Culicidae II. Beijing Science Press 1997, 9

37. Ma YJ, Qu FY, Xu JJ: Sequence differences of rDNA-ITS2 and species-diagnostic PCR assay of Anopheles sinensis and Anopheles anthropophagus from China. J Med Coll PLA 1998, 13:123-128.

38. HALL TA: Bioedit: a user-friendly biological sequence alignment editor and analysis program for windows 95/98/NT. Nucl. Acids Symp. Ser. 1999, 41:95-98.

39. Nei M: Molecular evolutionary genetics. New York: Colombia University Press; 1987.

40. Rozas J, Sanchez-Delbarrio J, Anchez-Delbarrio J, Messeguer X, Rozas R: Dnasp: DNA polymorphism analyses by the coalescent and other methods. Bioinformatics 2003, 19:2496-2497.

41. Tajima F: Statistical method for testing the neutral mutation hypothesis by DNA polymorphism. Genetics 1989, 123:585-595.

42. FU YX LIWH: Statistical tests of neutrality of mutations. Genetics 1993, 133:693-709.

43. Slatkin M: A measure of population subdivision based on microsatellite allele frequencies. Genetics 1995, 139:457-462

44. Weir BS, Cockerham CC: Estimating F-statistics for the analysis of population structure. Evolution Int J Org Evolution 1984, 38:1358-1370.

45. Schneider S, Excoffier L: Estimation of past demographic parameters from the distribution of the pariwise differences when the mutation rates vary among sites: application to human mitochondrial DNA. Genetics 1999, 152:1079-1089.

46. Excoffier L, Laval G, Schneider S: Arlequin (version 3.0): an integrated software package for population genetics data analysis. Evol Bioinform Online 2005, 1:47-50.

47. Rogers $A R$, Harpending $H$ : Population growth makes waves in the distribution of pairwise genetic differences. Mol Biol Evol 1992, 9:552-569.

48. FU YX: Statistical tests of neutrality of mutations against population growth, hitchhiking and background selection. Genetics 1997, 147:915-925.

49. Mantel N: The detection of disease clustering and a generalized regression approach. Cancer Res 1967, 27:209-220

50. Clement M, Posada D, Crandall KA: TCS: a computer program to estimate gene genealogies. Mol Ecol 2000, 9(10):1657-1660

51. Templeton AR, Crandall K, Sing CF: A cladistic analysis of phenotypic associations with haplotypes inferred from restriction endonuclease mapping and DNA sequence data. III. Cladogram estimation. Genetics 1992, 132:619-633.

52. Jung J, Jung Y, Min GS, Kim W: Analysis of the population genetic structure of the malaria vector Anopheles sinensis in South Korea based on mitochondrial sequences. Am J Trop Med Hyg 2007, 77:310-315.

53. Yang M, Ma Y, Wu J: Mitochondrial genetic differentiation across populations of the malaria vector Anopheles lesteri from China (Diptera: Culicidae). Malar J 2011, 10:216.

54. Sarma DK, Prakash A, O'Loughlin SM, Bhattacharyya DR, Mohapatra PK, Bhattacharjee K, Das K, Singh S, Sarma NP, Ahmed GU, Walton C, Mahanta J: Genetic population structure of the malaria vector Anopheles baimaii in north-east India using mitochondrial DNA. Malar J 2012, 11:76.

55. Conn JE, Vineis JH, Bollback JP, Onyabe DY, Wilkerson RC, Póvoa MM: Population structure of the malaria vector Anopheles darlingi in a malaria-endemic region of eastern Amazonian Brazil. Am J Trop Med Hyg 2006, 74:798-806.

56. Scarpassa VM, Conn JE: Population genetic structure of the major malaria vector Anopheles darlingi (Diptera: Culicidae) from the Brazilian Amazon, using microsatellite markers. Mem Inst Oswaldo Cruz 2007, 102:319-327.

57. Mirabello L, Vineis JH, Yanoviak SP, Scarpassa VM, Póvoa MM, Padilla N, Achee NL, Conn JE: Microsatellite data suggest significant population structure and differentiation within the malaria vector Anopheles darlingi in Central and South America. BMC Ecol 2008, 8:1472-6785.

58. Molina-Cruz A, de Merida AM, Mills K, Rodriguez F, Schoua C, Yurrita MM, Molina E, Palmieri M, Black IVWC: Gene flow among Anopheles albimanus populations in Central America, South America, and the Caribbean assessed by microsatellites and mitochondrial DNA. Am J Trop Med Hyg 2004, 71:350-359.

59. Michel AP, Guelbeogo WM, Grushko O, Schemerhorn BJ, Kern M, Willard MB, Sagnon N'F, Costantini C, Besansky NJ: Molecular differentiation between chromosomally defined incipient species of Anopheles funestus. Insect Mol Biol 2005, 14:375-387.
60. Michel AP, Grushko O, Guelbeogo WM, Lobo NF, Sagnon N'F, Costantini C, Besansky NJ: Divergence with gene flow in Anopheles funestus from the Sudan savanna of Burkina Faso, West Africa. Genetics 2006, 173:1389-1395.

61. Dong XS, Zhou HN, Gong ZD, Dong LM, Wang XZ: Investigation of mosquito species in Yunnan Province with some new species. Chin J Vector Biol Control 2004, 5:186-188.

62. Jin $\mathrm{ZZ}, \mathrm{Ou} X \mathrm{~K}$ : The diversity features of plant community types in the tropical rain forest vegetation of Xishuangbanna, Yunnan. Acta Bot Yunnan Suppl 1997, 1:1-30.

63. Zhu H, Xu ZH, Wang H, Li BG: Tropical rain forest fragmentation and its ecological and species diversity changes in southern Yunnan. Biol Conserv 2004, 13:1355-1372.

64. Liu X, Liu Q, Guo Y, Jiang J, Ren D, Zhou G, Zheng C, Liu J, Chen Y, Li H, Li $\mathrm{H}$, Li Q: Random repeated cross sectional study on breeding site characterization of Anopheles sinensis larvae in distinct villages of Yongcheng City, People's Republic of China. Parasit Vectors 2012, 5:58.

65. Audley-Charles MG: Dispersal of Gondwanaland: relevance to evolution of the angiosperms. In Biogeographical Evolution of the Malay Archipelago. Edited by Whitemore TC. Oxford: Clarendon Press; 1987.

66. Ng'habi KR, Knols BG, Lee Y, Ferguson HM, Lanzaro GC: Population genetic structure of Anopheles arabiensis and Anopheles gambiae in a malaria endemic region of southern Tanzania. Malar J 2011, 10:289.

67. Ballard JWO, Whitlock MC: The incomplete natural history of mitochondria. Mol Ecol 2004, 13:729-744.

doi:10.1186/1756-3305-6-290

Cite this article as: Makhawi et al: Genetic variations of ND5 gene of mtDNA in populations of Anopheles sinensis (Diptera: Culicidae) malaria vector in China. Parasites \& Vectors 2013 6:290

\section{Submit your next manuscript to BioMed Central and take full advantage of:}

- Convenient online submission

- Thorough peer review

- No space constraints or color figure charges

- Immediate publication on acceptance

- Inclusion in PubMed, CAS, Scopus and Google Scholar

- Research which is freely available for redistribution 